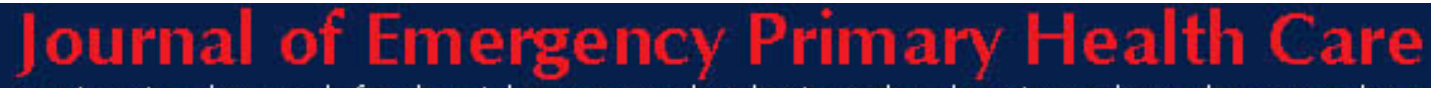

An International elournal of Prehospital Care Research, Education, clinical Practice, Policy and Service Delivery

ISSN 1447-4999

\title{
EDUCATION
}

Article 990192

\section{Using creative and contemporary teaching strategies to promote emancipation, empowerment and achievement in undergraduate paramedic students - a personal reflection}

\author{
Brett Williams \\ Lecturer, Centre for Ambulance and Paramedic Studies, Monash University, Melbourne
}

\section{Introduction:}

The case-based learning (CBL) process used in the clinical units of the Bachelor of Emergency Health - Paramedic (BEH-P) degree embraces similar ideologies to student-centred learning (SCL) and focuses on specific attributes such as: an increased search for independent knowledge, self-motivation, emancipation and self-discovery. The author has created greater student-to-student and student-to-teacher communication opportunities via several innovative strategies during each weekly case lecture series. These improvements have provided the opportunity for students to achieve their full potential whilst preparing them for the journey of a lifetime learner.

\section{Background:}

Contribution is specifically targeted to facilitate learning using CBL, SCL and online teaching and learning (T\&L) principles. This paper will present T\&L innovations founded upon student evaluations and critical reflection based on two clinical units from the BEH-P program: BEH2031 Foundations of Paramedic Clinical Practice and BEH2012 Paramedic Management of Cardio-Respiratory Emergencies. This is also emphasised with the recent thesis entitled "What are paramedic undergraduate students' perceptions of a clinical curriculum utilising a case-based learning paradigm?”

These contemporary approaches (online and CBL) have many similarities to traditional faceto-face (F2F) teaching; however there are particular nuances that require teachers to be cognizant of and make the appropriate alterations to their practice. ${ }^{1}$ Using CBL changes the passive learner to one who is self-directed and takes responsibility for his or her own education. Therefore, the role of a teacher must change from that of the authoritarian expert to coach or facilitator. ${ }^{2,3}$ The shift occurs from content-driven presentations to shared enquiry between learners and teachers in order to answer questions and solve problems. Educators must guide the discussion and remark on the learner's responses and summarise the content at the end of the learning segment rather than dominate the discussion with his or her own agenda or personal views. ${ }^{4}$

Within the BEH-P degree the clinical units use a CBL method in a blended T\&L approach with F2F lectures and online learning via WebCT (Vista). Real time or 'live' communication strategies are used to broaden student experiences. Using an e-learning approach allows the author to provide an interesting and interactive way of presenting realistic clinical cases 
(Figure 1) although in misguided hands e-learning can be hazardous to one's teaching approach, and consequently has been referred in the literature as 'Digital Hemlock' .

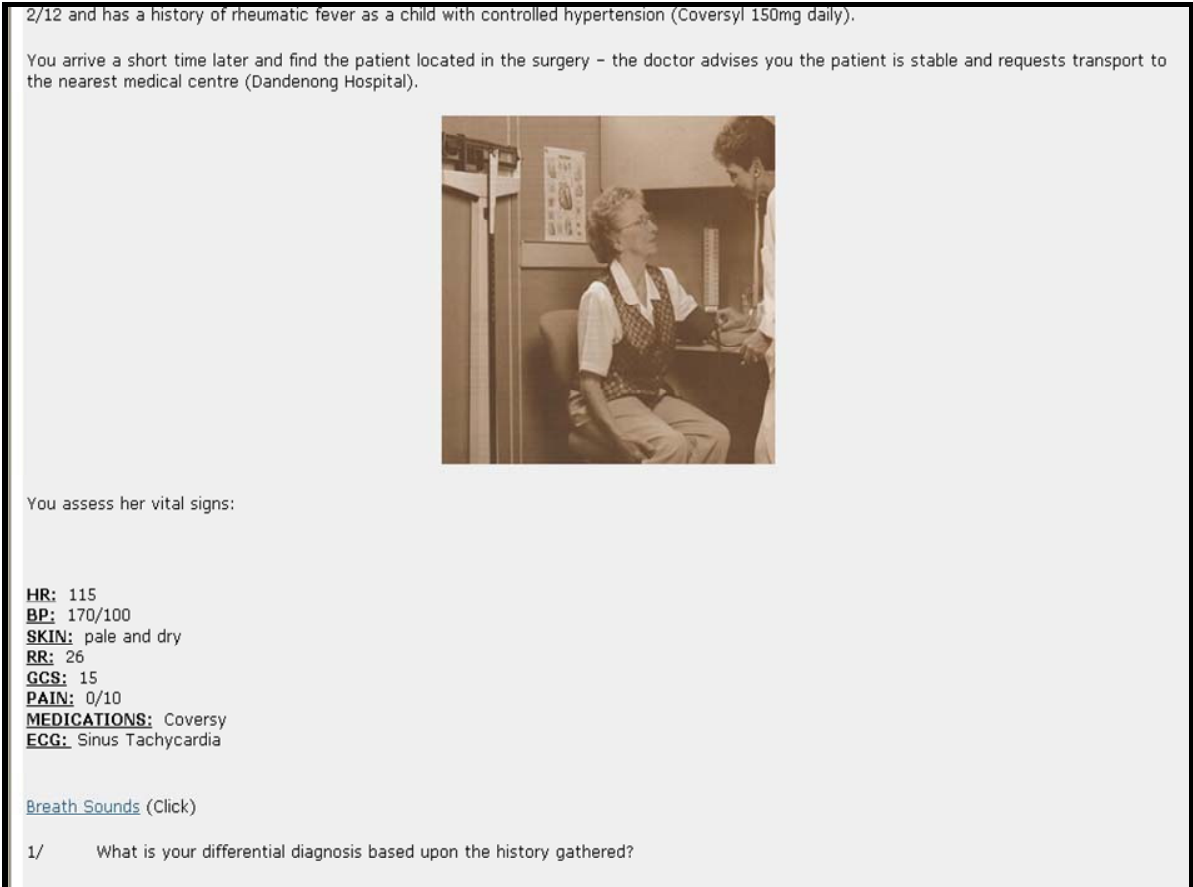

Figure 1. Example of realistic clinical case

The CBL framework used by the author has been adapted and modified from the Maastricht PBL ‘Seven-Jump’ Process (demonstrated visually below).

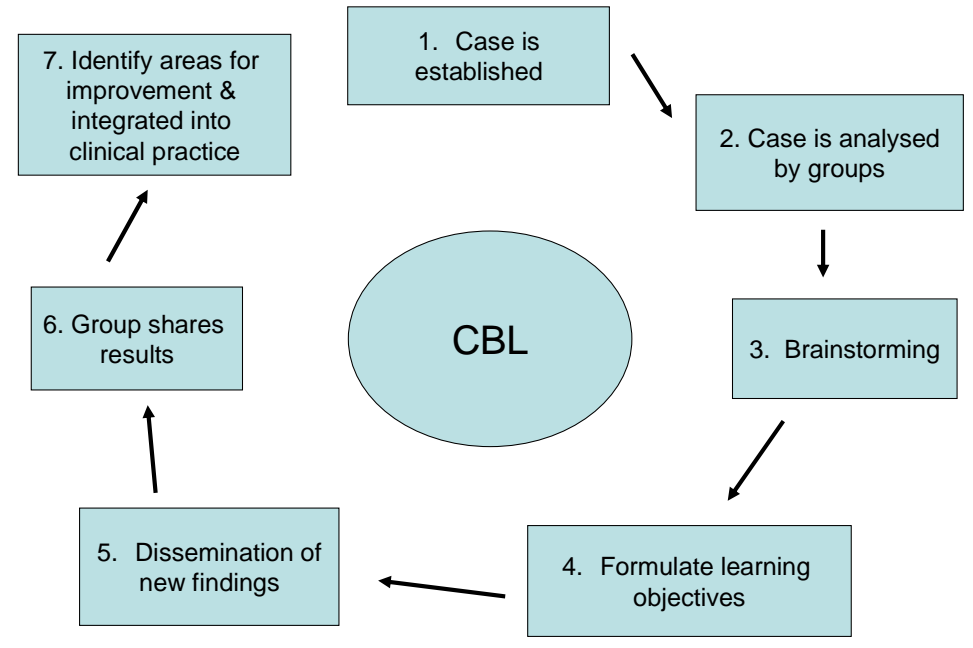

Figure 2. The CBL process (adapted from Maastricht PBL 'Seven-Jump’ process). ${ }^{6-8}$

Contribution to the CBL approach includes writing authentic clinical scenarios that introduce students to a patient with a clinical crisis, this reinforces the notion of CBL/SCL and enhances clinical praxis. ${ }^{1,2,9}$ Further information is provided such as: medical literature (supportive and controversial), vital signs, clinical manifestions and laboratory results. 
Presentation of the cases is presented in two forms: HTML and via WebCam to each etutorial group. ${ }^{6-8}$

\section{WebCam Example}

Patient details are provided in a variety of forms such as: text, audio files images and movie files. Each case is dissected by students within designated e-tutorial groups. Final analysis is provided by the author on the following Monday - this is generally a 10-15 minute summary of the previous weeks case. This ensures that all unanswered questions (and new questions) are answered prior to the commencement of a new week and case.

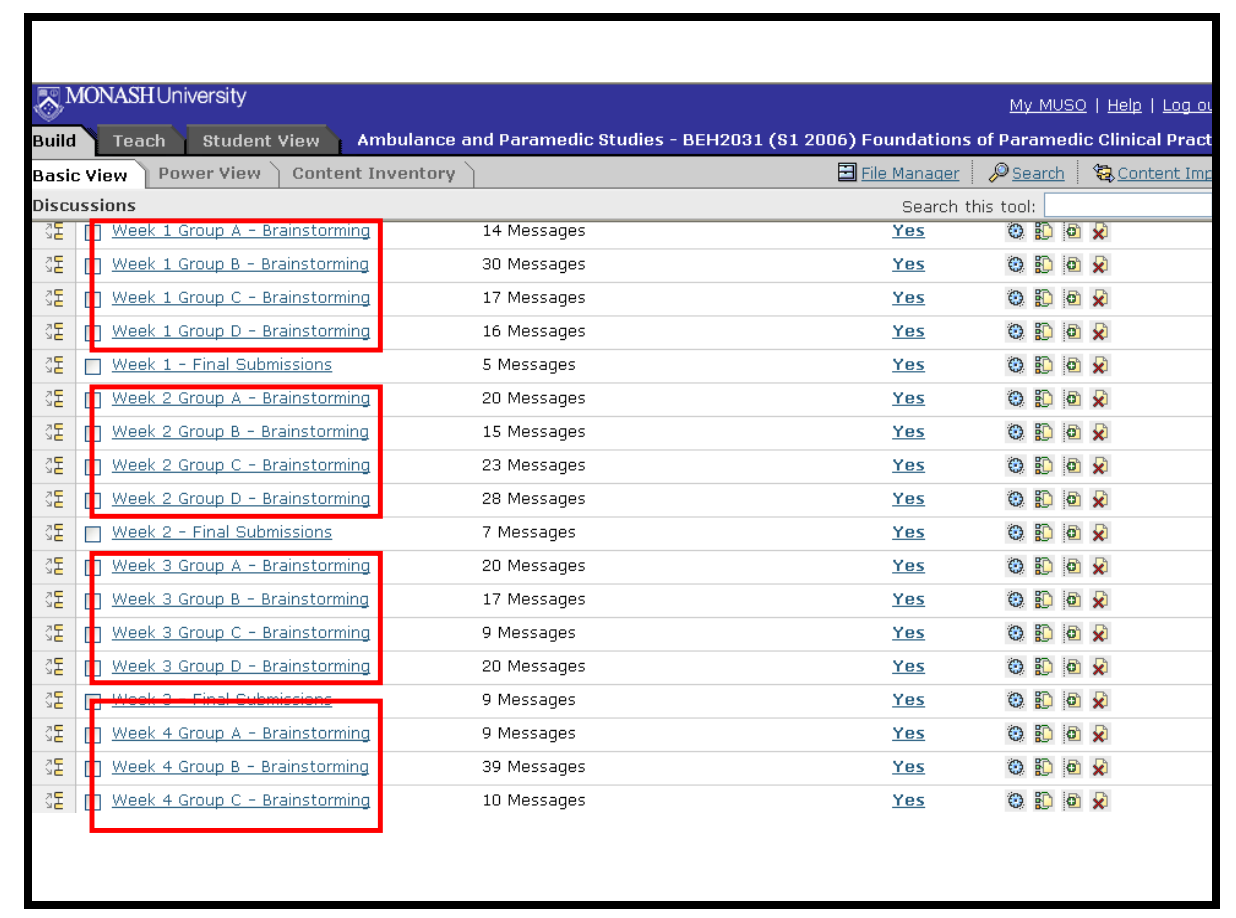

Figure 3. Example of e-tutorial groups

I believe our cognitive development has direct correlation to the social interactions and group learning dynamics. I place emphasis, time and attention to getting the tutorial groups right. The literature balances between the harmful and encouraging aspects of student and group dynamics in relation to SCL. ${ }^{3,10,11}$ Papers by Dolmans et al.;0a Visschers-Pleijers et al ${ }^{11}$ state that no evidence exists with regard to PBL and dysfunctional groups, however significant amounts of literature ${ }^{10-15}$ is available on difficult groups described by teaching staff that includes a lack of cooperation amongst students and an ambivalence with whom they should develop learning group partnerships. Debate, assimilation, elaboration are encouraged by case-based group learning in this environment. At the commencement of semester students are asked to identify what characteristics are considered important to work effectively as team members. The teaching intention is to draw attention to the key attributes they highlight to guide learning within the context of group dynamics. These attributes are then converted to 'team rules' for each e-tutorial group. The rules are uploaded within WebCT for students to refer to at any stage, or for the author to draw attention to when required. Interestingly, the paper by Silk et $\mathrm{al}^{1}$ also used similar "ground rules" in their T\&L approach in an e-learning context. The BEH rules were formulated by each separate tutorial group in the first week of semester, and were subsequently posted on WebCT. The student groups could view these rules at any time, examples included: 


\section{"Challenge each other" (Group A) \\ "Embrace constructive criticism” (Group B) \\ "Incorporate everyone’s ideas and opinions” (Group C) \\ “All questions and ideas are valid” (Group D)}

Each group is quarantined from the discussions of other e-tutorial groups, allowing each group to have private discussions amongst themselves. This strategy has been based on evaluation and reflection on previous experiences where student saturation of questions occurred on a regular basis, and identified by students as a distraction to their learning.

Using WebCT as the basis for my T\&L context offers students improved opportunities to achieve course learning objectives, thereby achieving my stated planned outcomes and more broadly, the overall syllabus intention. Students use this area to brainstorm to create fascinating and relevant discourse and to 'bounce' ideas of each other and lecturing staff. This is a crucial point, as learning is often an unplanned experience and has no clear learning framework. ${ }^{3}$ These discussion postings are used in formative and summative examinations, reinforcing the learning outcomes on a weekly basis via each case. The use of WebCT's online exam feature allows the assessment of learning growth and needs from a web-based exam perspective.

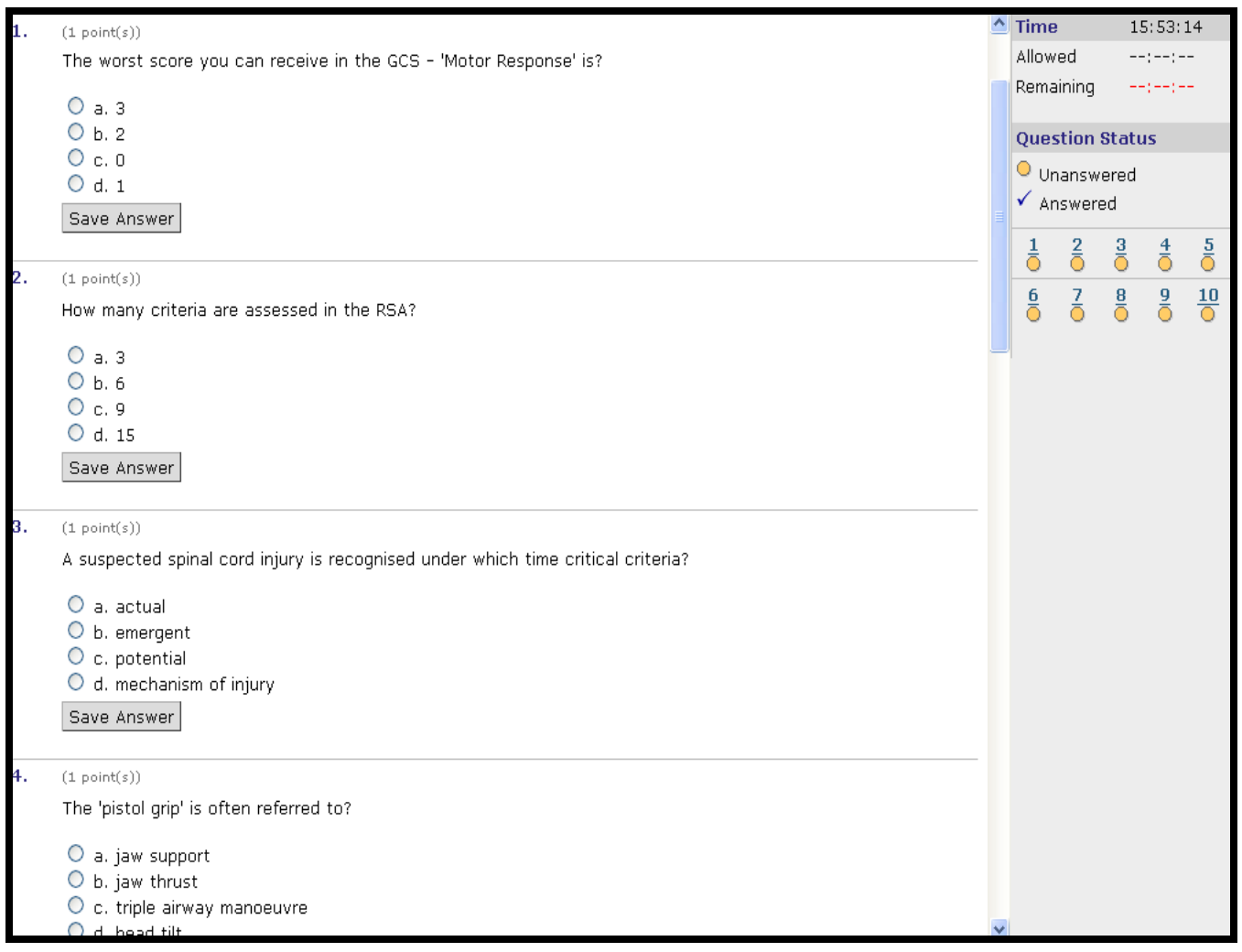

Figure 4. WebCT examination questioning area highlighting the multiple-choice questioning layout.

Guiding this T\&L approach are educational theories based upon the belief that students are responsible for their learning and that significant learning occurs within contexts of social 
learning groups. ${ }^{16-19}$ The author's philosophical approach allows students to drive their learning needs and outcomes as I take the stance of a co-learner. My passion and motivation to teach well and to inspire my students to 'cognitively challenge' themselves is based upon this notion of a co-learner. Pragmatism and critical reflection is undertaken on a daily basis - on what each student expects from my sessions, this is done from the view that I am a student of learning, that learning is just that: learning, therefore should be fun regardless of what subject matter is being taught. It is my intention to not only challenge and motivate my students but it should be done in a non-threatening and respectful environment. An important facet of this approach is the notion that I am opposed to being simply a 'knowledge dispenser'.

\section{Approaches and Innovations:}

Online education involves and requires generally high student motivation and discipline. ${ }^{5}$ These are core attributes of CBL and SCL programs; accordingly, this creates a synergism whereby the self-directed learning is crucial to not only participate in the e-learning but also to develop correct clinical answers to the case. This self-directedness is also enhanced by the learning undertaken at the student's own pace and is central in andragogy (adult learning) whereby students relate learning to past experiences as an active learner. ${ }^{3}$ A review of the students' discussion postings indicated that many of these responses were undertaken outside 'normal' working hours and also on weekends see Table 1.

\begin{tabular}{|c|c|}
\hline Total user sessions & 4318 \\
\hline Average user session length & $0: 10: 27$ \\
\hline Average user sessions per day & 53 \\
\hline $\begin{array}{c}\text { Average user sessions per day on } \\
\text { weekdays }\end{array}$ & 72 \\
\hline $\begin{array}{c}\text { Average user sessions per day on } \\
\text { weekends }\end{array}$ & 38 \\
\hline Most active hour of the day & $1300-1400 \mathrm{hrs}$ \\
\hline Least active hour of the day & $0400-0500 \mathrm{hrs}$ \\
\hline
\end{tabular}

Table 1. Second year WebCT activity log semester 1.

Analysis of research data and MonQuest evaluation forms highlighted that the flexible online environment was a beneficial place to learn:
"Doing the work online meant I could fit in the tute work with other work commitments" (MonQuest - Student [32] BEH2021)
"Online was good as I could also search for info on websites relating to the topic" (MonQuest - Student [12] BEH2031)

However, other comments from students highlighted for them, the online environment was not necessarily accommodating or beneficial for their learning:

"As I do not have access to the internet at home during the week it was often difficult to participate” (Thesis - Student [9] BEH2031) 
"Often found it difficult to be online" (Thesis - Student [13] BEH2021)

Analysis of qualitative data results also demonstrated that the CBL blended process was overwhelming enjoyed by students.

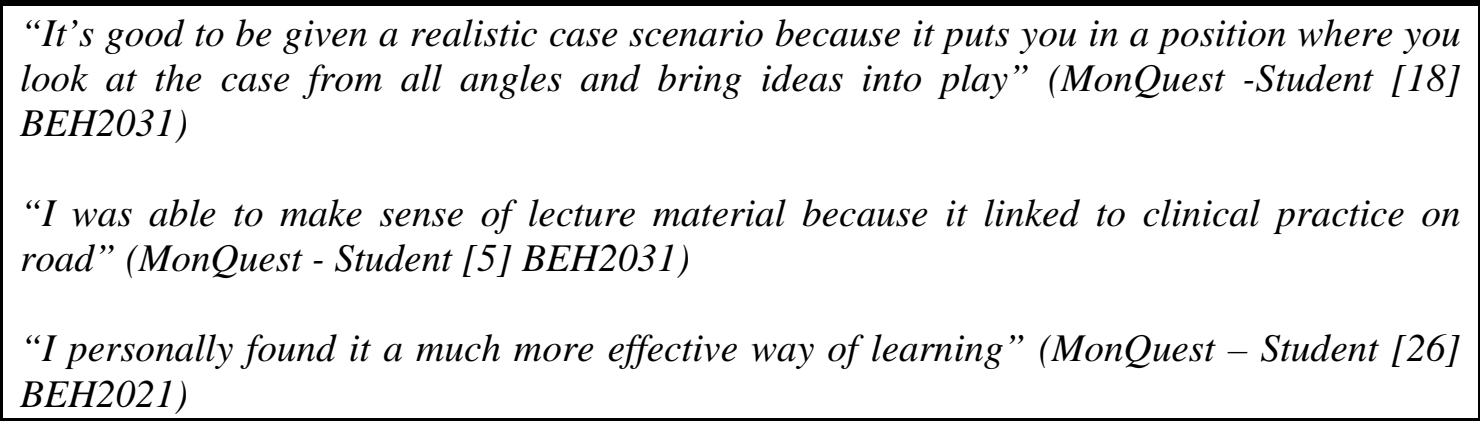

Web accessibility is an important part of my teaching philosophy, practice and quality assurance. The following are examples of my approach in this context:

\section{Student Needs Analysis}

The commencement of each study year brings a broad range and variety of learners. To ensure that my teaching remains multidimensional, a student needs-analysis is undertaken of learner generation and information technology (IT) capacity prior to the commencement of each teaching period. This provides me with flexibility to adjust my T\&L approach, particularly if the student group is less confident with web-based education.

At the start of semester analysis is provided on the following:

Age: Since our students continue to come from a range of generations. Learning generations have been commonly defined in the following terms, Net Generation (24 years and younger), Generation X (24 to 44 years), and Boomers (44 to 64 years). ${ }^{20}$ It is essential for teachers to meet the students learning needs and address potential learning obstacles such as computerbased education. $^{2}$

Location: Knowing the student geographic locations allows me to to 'map' the student cohort and identify potential learning disadvantages for example, distance to campus and capacity to podcast guest lecture material or F2F lecture material, that could be listened in the car, tram or train. In other words, better utilising their study time whilst also examining the notion of spatial learning.

Internet Access: Establishing if students have Internet access at home is crucial, particularly for units using a blended approach, since the very success of study will require students to undertake WebCT discussion postings and/or emails whilst at home. Also, the type of Internet access (cable, ADSL, dial-up) is important, for example, if a student only has dialup then consideration must be given to dial-up and download speed. Analysing these learning needs and requirements, provides students and the author with clear setting of what difficulties may be expected and consideration of altering elements of the T\&L approach. In other words, if $40 \%$ of students only have Internet at home, then the process of CBL used in a blended approach will simply fail.

\section{CD-ROM:}


Regular evaulation and monitored use of WebCT ensures the T\&L environment is kept concise with easy navigation and responsive IT support. The author has developed the first BEH-P student resource CD-ROM, created to assist students with required software, allowing a significant reduction in download times and better use of learning time. Importantly, this CDROM can be used in the student's clinical placement time, again highlighting the importance of ensuring students utilise their learning time effectively.

\begin{tabular}{|c|c|}
\hline 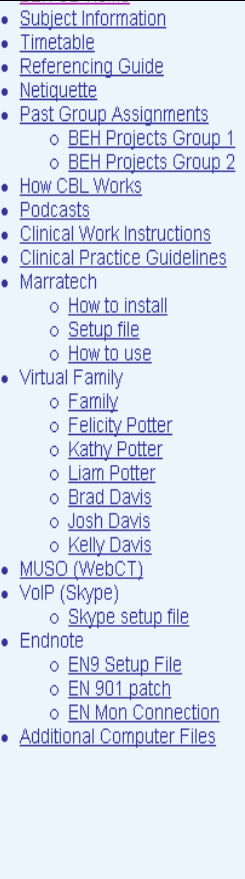 & $\begin{array}{l}2006 \text { Resources CD - and files for BEH2031 } \\
\text { INTRODUCTION } \\
\text { This CD-ROM contains various files that will be required during the semester for BEH2031 and a Java Runtime } \\
\text { that has been verified to work correctly with WebCT Vista. } \\
\text { It also contains additional software that may be required to view or access content on WebCT Vista. } \\
\text { DISCLAIMER } \\
\text { Monash University accepts no responsibility for any loss or darnage or consequential loss or damage, arising } \\
\text { from the use of this CD-ROM. This includes loss of data or interference with files arising from the use of } \\
\text { software on this CD-ROM. } \\
\text { Installation Instructions } \\
\text { In order for all features of MUSO (WebCT Vista) to work correctly you will need: } \\
\text { A. a compatible web browser that is configured correctly (preferably Internet Explorer) } \\
\text { B. the Java Runtime software installed and enabled. }\end{array}$ \\
\hline
\end{tabular}

Figure 5. BEH-P Student Resource CD Rom

\section{Virtual Family}

This semester has seen the creation of a 'virtual family' - which consists of the Potter and Davis families (Figure 6).

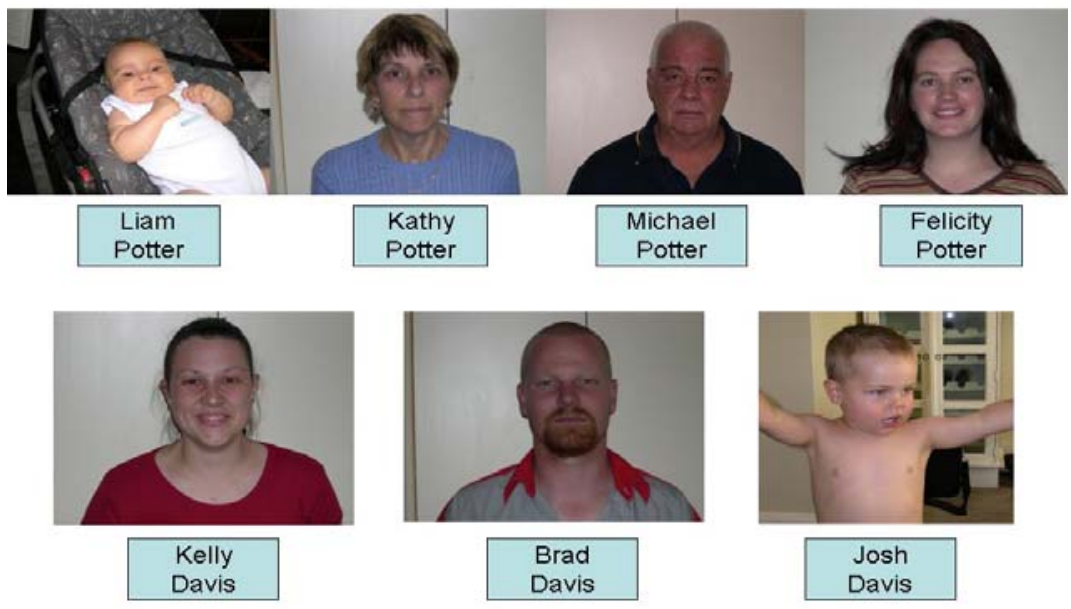

Figure 6. Virtual Family 
All cases throughout the semester are based upon the family members, who have predesignated medical, social histories and specific physiological parameters within a 'history sheet'. The history sheet (Figure 7) has specific history items that directly link to the weekly case - the goal is that implicit or hidden questions/thoughts will be raised (including the explicit questions) by students that relate to the other themes within the BEH-P degree.

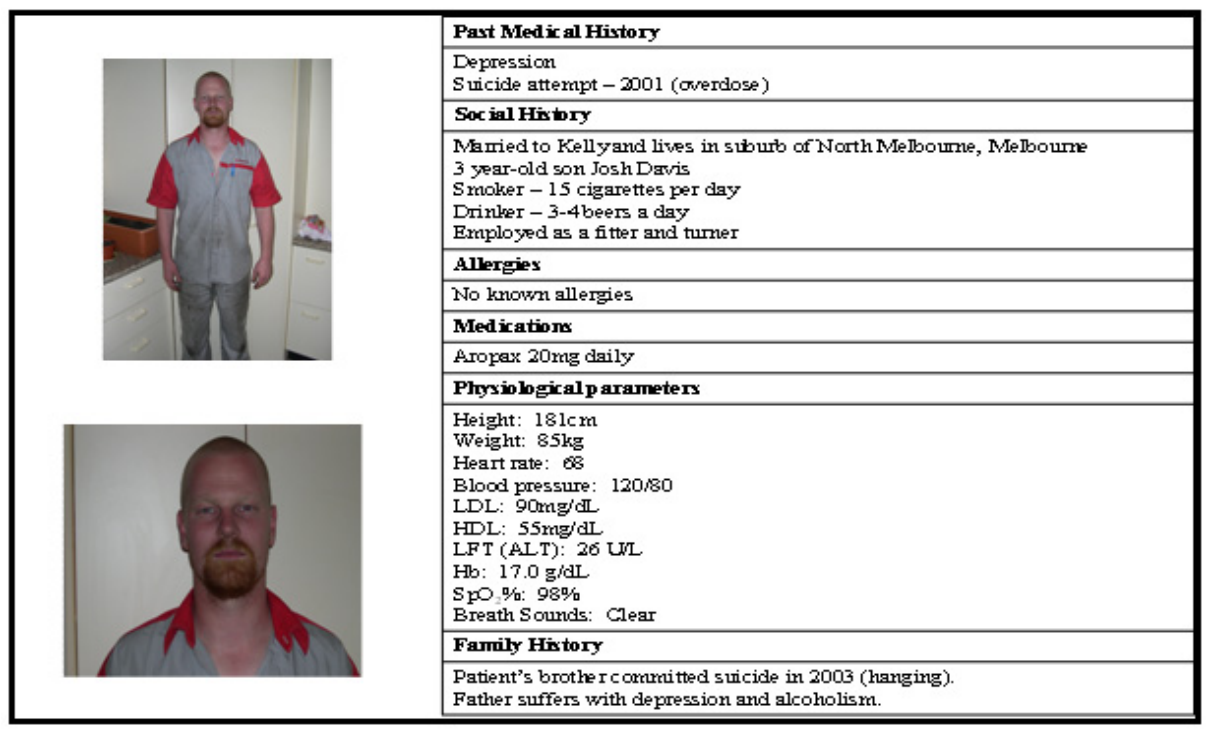

Figure 7. Example of History Sheet

Each case throughout the semester uses one of the family members in different clinical contexts. For example, Kathy Potter has shortness of breath secondary to smoke inhalation in week 4 and has a collapse due to tachycardia-bradycardia syndrome in week 10 . The educational intention is to draw students to each family member in a more familiar context, encouraging them to focus on the key points in their history sheets regardless of what clinical crisis they are placed in.

\section{Podcasts}

Podcasting was first created in 2000 and is now considered a technological phenomenon, although presently it is sparingly used in the higher education sector. ${ }^{21}$ A podcast is a file (photo, music or movie) that can be recorded and broadcast (podcast) via the Internet to listeners in real-time or asynchronously (delay). ${ }^{21}$ The clinical units in the BEH-P program are offered 'Podcast Guest Lecture Series' that is designed to introduce students to the concepts from experts working in the prehospital/community-based emergency health care. Podcasts are scheduled online during the teaching period as an integral and important part of the BEH-P program, with guest lecturers addressing the student group on a range of topics broadly relevant to the overall learning objectives and goals of the BEH-P, current evidence and literature. Podcasts are offered as a reinforcing learning tool for students. They are recorded from a personal office and converted to audio files (i.e. MP3) that can be listened to in a variety of ways and, in a variety of settings at the pace of each student learner, a true ondemand control. ${ }^{21}$ This provides the students with the ability to listen to audio material on the train, in the library or at home as many times as they like. 


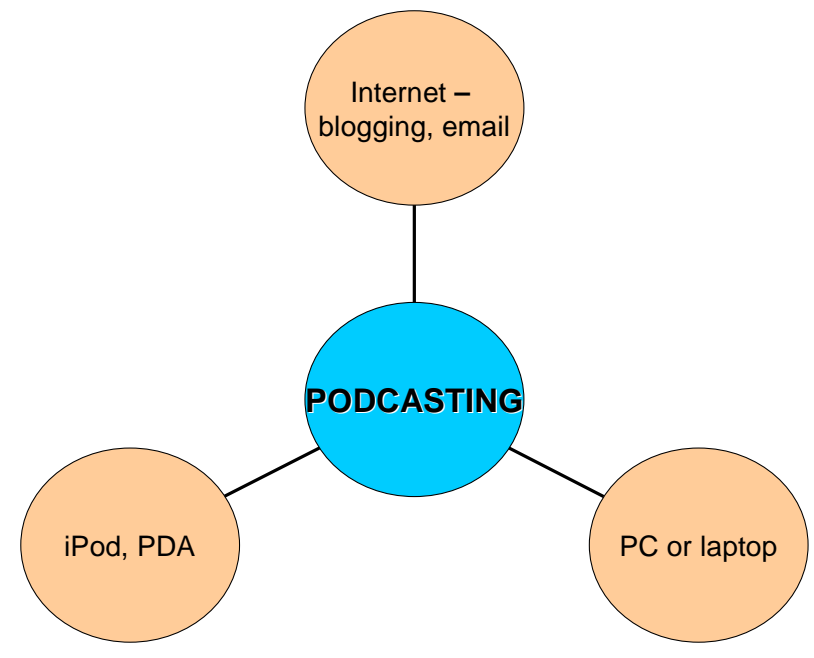

Figure 8. Integration of Podcasts

\section{Example of Podcast (requires media player)}

\section{Voice over Internet Protocol (VoIP)}

Voice over Internet Protocol (VoIP) is a revolution in telecommunications that allows clients to make telephone calls using a broadband Internet connection instead of a regular phone landline. This type of synchronous (real-time) communication is a relatively new technology that offers great T\&L opportunities for teachers and students. ${ }^{22}$ BEH-P students are offered the opportunity to communicate with the author via Skype (Figure 9). Skype is peer-to-peer VoIP first developed by $\mathrm{KaZaa}^{23}$ that has capacity for instant messaging, audio conferencing, allows users to talk one-on-one or multiple people, send chat messages and receive view live streaming movie files. Another innovation has been the implementation of real-time communication strategies. This software allows students to call me in designated working and after hours. Providing students with timely feedback is critical in my teaching practice, particularly in the e-learning environment where students often feel isolated and frustrated by lengthy delays in teacher responses. ${ }^{1}$

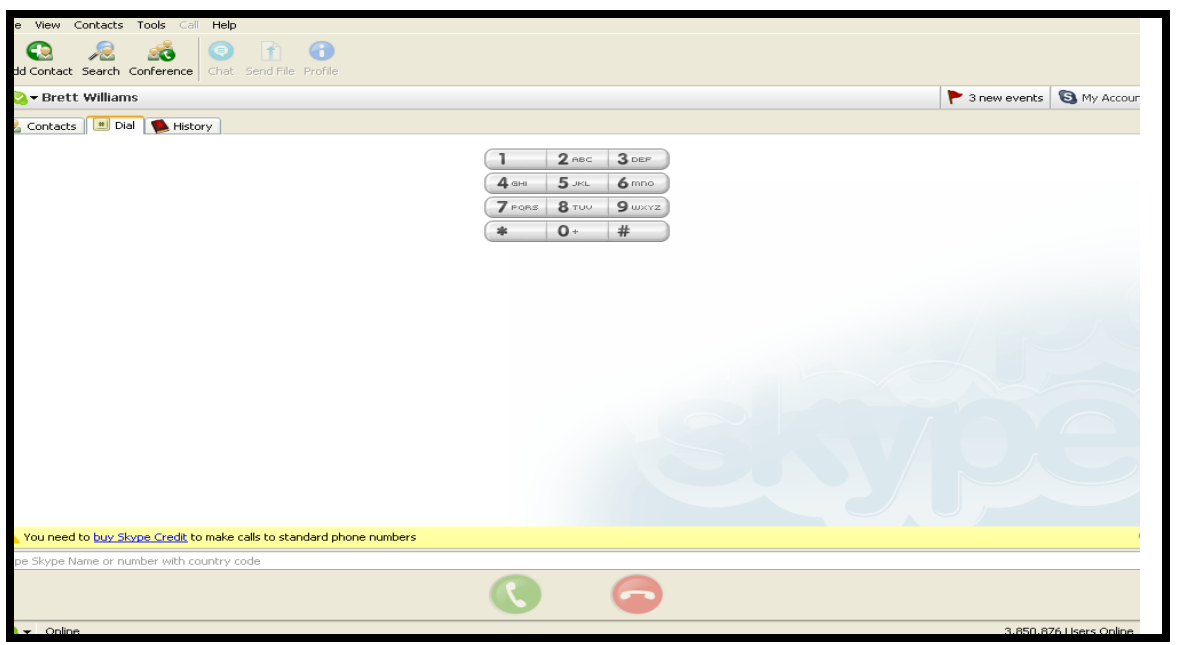

Figure 9. Example of Skype 


\section{Internet Videoconferencing}

Internet desktop videoconferencing, allows participants working from a computer, to send videos (webcam) and audio (headset or microphone) to other participants (students and/or teaching staff). The software currently being trialled by Monash University for online conferencing sessions is called "Marratech", although other software programs exist (Elluminate, Macromedia Breeze and Mediasite). Internet videoconferencing has been recently introduced in medical education with several papers suggesting this type of teaching is not only cost-effective but also an acceptable method of teaching medical students. ${ }^{24,25}$ Marratech is an international web conference provider that provides teaching facilities such as Universities in its desktop video conferencing facility. This allows a collaborative approach between teachers and students in 'real-time' without frustrating delays. The Marratech Portal runs on a central server and the Marratech client is downloaded on student's computers. The Marratech portal runs continuously so students and lecturers can talk to each other at any time. Two sessions a week are offered to students on Tuesday afternoons and Thursday mornings, however it would be possible for student-to-student or student-teacher to organise meetings not just in scheduled online tutorial sessions. Lastly, the integration of such a software program offers real-time audio with chat and video file features. A key feature allows the integration of PowerPoint slides with blackboard capabilities. This feature allows me to draw, highlight and emphasise the lecture PowerPoint's with students. Descriptive evaluation will be undertaken on this T\&L resource to assess students' experiences and attitudes using Marratech next semester.

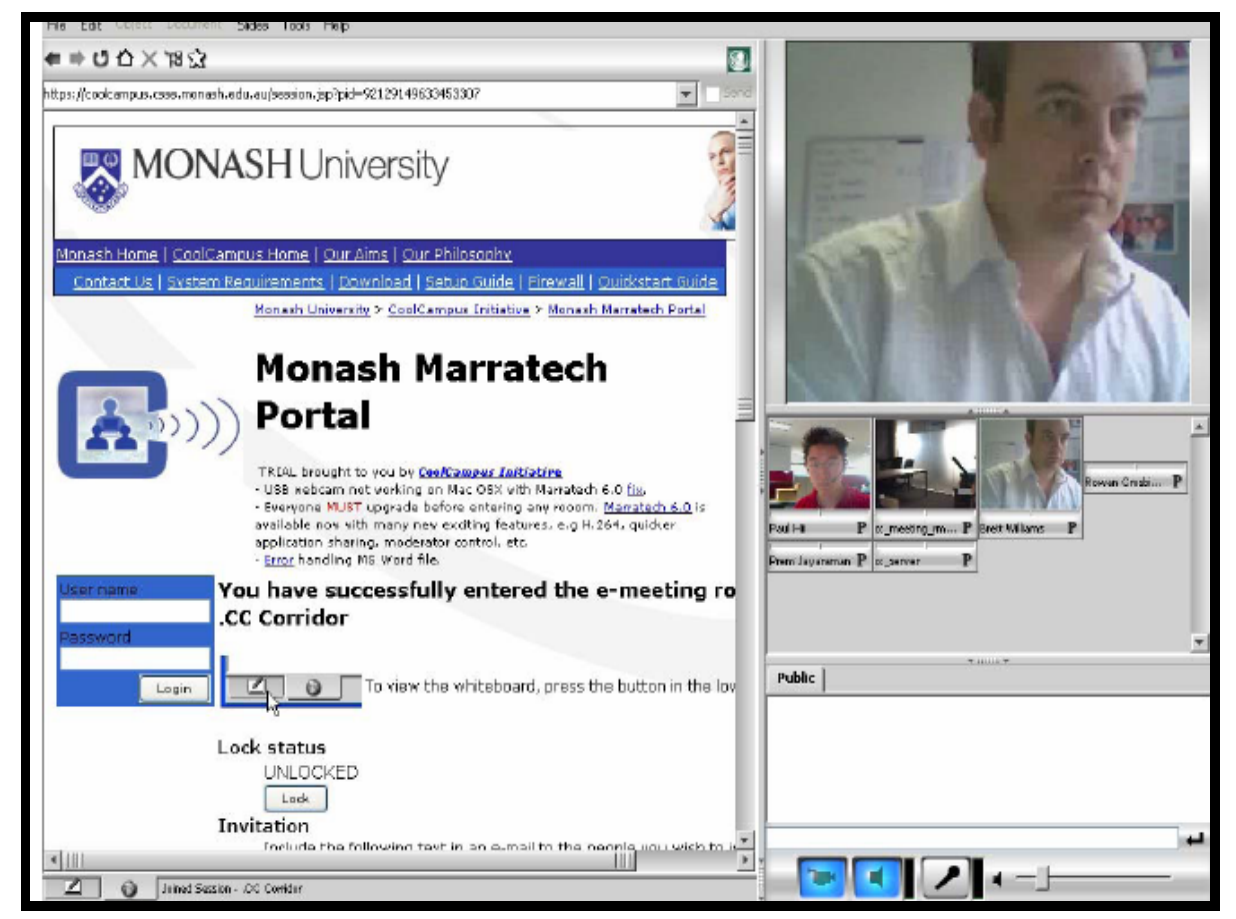

Figure 10. Example of Marratech Portal

\section{Conclusion:}

This paper has attempted to draw upon the author's T\&L landscape along with experiences and promising teaching innovations according to a student-centred approach. Positive student evaluations have suggested these approaches are, and have, supported the majority of BEH-P students in their learning. These web-based innovations however, are not undertaken 
frivolously given the educational climate in the higher education sector regarding online education. Consequently, the author's teaching approaches have strong support and have clear convincing educational rationale based upon committed teaching critical reflection and reflexivity. As the ergonomics of higher education learning continues to shift, respect and caution must be given to Internet-based education, or as Tara Brabazon puts it ... "digital disaster” is just around the corner! 


\section{References:}

1. Silk H, Agresta T, Weber C. A New Way to Integrate Clinically Relevant Technology into Small-Group Teaching. Academic Medicine 2006;81(3):239-244.

2. Cook D, Thompson W, Thomas K, Thomas M, Pankratz S. Impact of Self-Assessment Questions and Learning Styles in Web-Based Learning: A Randomised, Controlled, Crossover Trial. Academic Medicine 2006;81(3):231-238.

3. Ruiz J, Mintzer M, Leipzig M. The Impact of E-Learning in Medical Education. Academic Medicine 2006;81(3):207-212.

4. Phillips JM. Strategies for active learning in online continuing education. Journal of Continuing Education in Nursing 2005;36(2):77-83.

5. Brabazon T. Digital Hemlock: Internet education and the poisoning of teaching. Sydney: UNSW Press; 2002.

6. Williams B. The Implementation of Problem-Based Learning \& Case-Based Learning: Shaping the Pedagogy in Ambulance Education - A MUCAPS Experience. In:

Proceedings of the Australian College of Ambulance Professionals (ACAP); 2004 Sept 911; Alice Springs; 2004.

7. Williams B. CBL for Paramedic Students. In: Higher Education Partnerships in Communication and Information Technology; 2005; Monash University; 2005.

8. Williams B. Student Perceptions of CBL by Paramedic Students. In: Vice Chancellor Teaching Showcase; 2005; Monash University; 2005.

9. Bowen D. Integrating case-based instruction into dental hygiene curricula. Journal of Dental Education 1998;62(3):253-256.

10. Dolmans DH, Wolfhagen IH, van der Vleuten CP. Why aren't they working? London: Kogan Page; 2001.

11. Visschers-Pleijers AJ, Dolmans DH, Wolfhagen IH, Van der Vleuten CP. Student perspectives on learning-oriented interactions in the tutorial group. Advances in Health Sciences Education 2005;10(1):23-35.

12. Huda N, Brula AQ. An introductory course on study skills forming a bridge between traditional and problem based learning (PBL). JPMA - Journal of the Pakistan Medical Association 1999;49(2):27-30.

13. Das Carlo M, Swadi H, Mpofu D. Medical student perceptions of factors affecting productivity of problem-based learning tutorial groups: does culture influence the outcome? Teaching \& Learning in Medicine 2003;15(1):59-64.

14. 14. Pang SM, Wong TK, Dorcas A, Lai CK, Lee RL, Lee WM, et al. Evaluating the use of developmental action inquiry in constructing a problem-based learning curriculum for pre-registration nursing education in Hong Kong: a student perspective. Journal of Advanced Nursing 2002;40(2):230-41.

15. Dolmans DH, De Grave W, Wolfhagen IH, van der Vleuten CP. Problem-based learning: future challenges for educational practice and research. Medical Education 2005;39(7):732-41.

16. Piaget J. Six Psychological Studies. New York: Vintage Books; 1968.

17. Bruner J. Toward a Theory of Instruction. Cambridge: Harvard University Press; 1966.

18. Dewey J. Demoracy and Education. An introduction to the philosophy of education. New York: Free Press; 1916.

19. Vygotsky L. Mind in Society. London: Harvard University Press; 1978.

20. Billings DM, Rowles CJ. Development of continuing nursing education offerings for the World Wide Web. Journal of Continuing Education in Nursing 2001;32(3):107-13.

21. Hagis J, Wilson D. Fishing for Learning with a Podcast Net. In; 2005.

22. Schullo S, Venable M. Synchronous E-learning: Proven Strategies for Teaching at a Distance. In; 2005. 
23. Baset SS, H. An Analysis of the Skype Peer-to-Peer Internet Telephony Protocol. In; 2004.

24. Misra UK, Kalita J, Mishra SK, Yadav RK. Telemedicine for distance education in neurology: preliminary experience in India. J Telemed Telecare 2004;10:363.

25. Cook A., Salle JL., Reid J., Chow KF., Kuan J., Razvi H., et al. Prospective evaluation of remote, interactive videoconferencing to enhance urology resident education: the genitourinary teleteaching initiative. Journal of Urology 2005;174(5):1958-60.

\section{Author Disclosure}

The author has no financial, personal or honorary affiliations with any commercial organization directly involved or discussed in this study. 